\title{
Alternativa restauradora por meio de endocrown: uma revisão da literatura
}

Restoring alternative through endocrown: a review of the literature

Alternativa restauradora por medio de endocrown: una revisión de la literatura

$$
\text { Monnyk Stefane Figueredo e SILVA }{ }^{1}
$$

José Henrique de Araújo $\mathbf{C R U Z}^{1}$

Daniela Lima CRISTINO ${ }^{1}$

Rodrigo Araújo RODRIGUES ${ }^{2}$

João Nilton Lopes de SOUSA ${ }^{2}$

Barbara Vanessa de Brito MONTEIRO²

Rodrigo Alves RIBEIRO ${ }^{2}$

${ }^{I}$ Acadêmico(a) do Curso de Graduação em Odontologia. Centro de Saúde e Tecnologia Rural, Universidade Federal de Campina Grande, UFCG 58708-110 Patos/PB, Brasil

${ }^{2}$ Professor(a) Doutor(a) do Curso de Graduação em Odontologia. Centro de Saúde e Tecnologia Rural, Universidade Federal de Campina Grande, UFCG 58708-110 Patos/PB, Brasil

\section{Resumo}

Introdução: A coroa endocrown é uma restauração indireta confeccionada em cerâmica adesiva em monobloco e cimentada com técnicas adesivas. A coroa possui como retenção a câmara pulpar do dente tratado endodonticamente sem que se utilizem os condutos. Dentes tratados endodonticamente possuem redução da rigidez e resistência devido à perda da integridade estrutural. Este fato pode acarretar em longo prazo falhas biomecânicas. Objetivo: Realizar uma revisão de literatura do tipo narrativa acerca das principais características das endocrowns. Metodologia: A pesquisa foi realizada no período de 20 de Janeiro a 02 de Abril de 2019. Foram utilizados artigos científicos das seguintes bases de dados: SCIELO (Scientific Eletronic Library Online), BVS (Biblioteca Virtual em Saúde), e monografias que atendiam aos requisitos no período entre 2008 a 2019, com exceção de artigos clássicos, em língua nacional, português, bem como em inglês e espanhol. Foram utilizados como descritores isolados, ou combinados, para a busca: "Endocrown", "Endocrown Prótese", "Endocrown Prosthesis", "Endocrown Prótesis", com um resultado de 31 artigos utilizados. Conclusão: As endocrowns são indicadas para dentes posteriores tratados endodonticamente, confeccionada na maioria das vezes por dissilicato de lítio e apresenta estudos que comprovem que o risco de fratura é inferior às reabilitações sob uso de retentores intrarradiculares. Sob boa indicação e avaliação, é possível obter previsibilidade de sucesso, porém, ainda são necessários mais pesquisas para comprovar cada vez mais a eficácia dessa alternativa.

Descritores: Prótese Dentária; Prótese Parcial Fixa; Odontologia.

\section{Abstract}

Introduction: The Crown endocrown is an indirect restoration made of adhesive monobloc ceramic and cemented with adhesive techniques. The crown retains the pulp chamber of the endodontically treated tooth without using the conduits. Endodontically treated teeth have reduced stiffness and resistance due to loss of structural integrity. This can lead to long-term biomechanical failures. Objective: To carry out a narrative literature review about the main characteristics of endocrowns. Methodology: The research was carried out from January 20 to April 2, 2019. Scientific articles were used from the following databases: SCIELO (Scientific Electronic Library Online), VHL (Virtual Health Library), and monographs that requirements in the period between 2008 and 2019, except for classic articles, in Portuguese, as well as in English and Spanish. We used the following descriptors for the search: "Endocrown", "Endocrown Prosthesis", "Endocrown Prosthesis", "Endocrown Prosthesis", with a result of 31 articles used. Conclusion: Endocrowns are indicated for endodontically treated posterior teeth, most often made by lithium disilicate, and present studies proving that the risk of fracture is inferior to the rehabilitations under the use of intraradicular retainers. Under good indication and evaluation, it is possible to obtain predictability of success, however, further research is needed to prove more and more the effectiveness of this alternative.

Descriptors: Dental prosthesis; Fixed Partial Prosthesis; Dentistry.

\section{Resumen}

Introducción: La corona endocrown es una restauración indirecta confeccionada en cerámica adhesiva en monobloque y cementada con técnicas adhesivas. La corona posee como retención la cámara pulpar del diente tratado endodónticamente sin que se utilicen los conductos. Dientes tratados endodónticamente poseen reducción de la rigidez y resistencia debido a la pérdida de la integridad estructural. Este hecho puede acarrear a largo plazo fallas biomecánicas. Objetivo: Realizar una revisión de literatura del tipo narrativa a cerca de las principales características de las endocrowns. Metodología: La investigación se realizó en el período de 20 de enero a 2 de abril de 2019. Se utilizaron artículos científicos de las siguientes bases de datos: SCIELO (Biblioteca Virtual en Salud), BVS (Biblioteca Virtual en Salud), y monografías que atendían a los siguientes: requisitos para el período de 2008 a 2019, con excepción de los artículos clásicos en el idioma nacional, portugués, así como Inglés y Español. Se utilizaron como descriptores aislados, o combinados, para la búsqueda: "Endocrown", "Endocrown Prótesis", "Endocrown Prosthesis", "Endocrown Prótesis", con un resultado de 31 artículos utilizados. Conclusión: Las endocrowns son indicadas para dientes posteriores tratados endodónticamente, confeccionada en la mayoría de las veces por disilicato de litio y presenta estudios que comprueban que el riesgo de fractura es inferior a las rehabilitaciones bajo uso de retentores intrarradiculares. Bajo buena indicación y evaluación, es posible obtener previsibilidad de éxito, sin embargo, todavía se necesitan más investigaciones para comprobar cada vez más la eficacia de esa alternativa.

Descriptores: Prótesis dental; Prótesis Parcial Fija; Odontología.

INTRODUÇÃO

A rigidez e resistência de dentes tratados endodonticamente são menores comparados aos dentes vitais, devido a problemas como a cárie, traumatismos e preparações extensas ou alargamento da câmara pulpar. Estas intercorrências podem acarretar em falhas biomecânicas, falta de elasticidade e resistência à tração de forças mastigatórias. Associado a isso, problemas mais graves como fraturas podem ocorrer ${ }^{1-4}$.

A reabilitação funcional e estética deve ser realizada com cautela, já que a longevidade do tratamento endodôntico é influenciada por ela, bem como pela adequada seleção do tipo de preparo que busque conservar o máximo de estrutura dentária ${ }^{1,3-5}$.

A alternativa para dentes com extensa perda coronária é a utilização de um núcleo metálico fundido ou pino pré-fabricado ${ }^{6}$, tendo maior eficácia os pinos pré-fabricados que possuem propriedades biomecânicas $^{7,4}$. Embora apresentem resultados satisfatórios, os preparos de coroa total realizam a remoção dos últimos remanescentes de tecidos coronários ao redor do acesso endodôntico, causando 
um enfraquecimento do elemento dental ${ }^{8}$.

Deste modo, tratamentos alternativos vêm sendo pesquisados para serem utilizadas como opções restauradoras, sendo a técnica endocrown uma delas. A coroa endocrown é uma restauração indireta confeccionada em cerâmica adesiva em monobloco e cimentada com técnicas adesivas, a coroa possui como retenção a câmara pulpar do dente tratado endodonticamente sem que se utilizem os condutos $^{2,3}$. A endocrown teve a sua primeira aplicação por volta de 1989 a 1992, variando a técnica de monobloco concluído com material de vitro-cerâmica ${ }^{4}$.

Atualmente existem diversos estudos relatando a utilização de coroas endocrown com uma boa taxa de sobrevivência das restaurações, reduzindo os riscos de complicações uma vez que o preparo interno fica limitado à cavidade pulpar, não causando a desobturação ${ }^{1,4,5,7,9}$.

Tendo em vista as aplicações e indicações das Endocrowns e por ser um tratamento reabilitador prático, objetivou-se realizar uma revisão da literatura acerca dos seus aspectos importantes diante do assunto.

\section{MATERIAL E MÉTODO}

Trata-se de revisão narrativa da literatura, ao buscar publicações amplas, apropriadas para descrever e discutir o desenvolvimento ou o "estado da arte" de um determinado assunto, sob ponto de vista teórico ou contextual e, dessa forma, na análise de publicações de livros, artigos de revista impressas e/ou eletrônicas, tecer interpretação e avaliação crítica pessoal do autor ${ }^{10}$. A pesquisa foi realizada no período de 20 de Janeiro a 2 de Abril de 2019. Com embasamento para a pesquisa, foram utilizados artigos científicos retirados das seguintes bases de dados: SCIELO (Scientific Eletronic Library Online), BVS (Biblioteca Virtual em Saúde) e PubMed monografias que atendiam aos requisitos do estudo em questão. Os artigos utilizados como referências correspondem ao período entre 2008 a 2019, com exceção de artigos clássicos necessários para a escrita do trabalho, do qual a pesquisa foi realizada tanto na língua nacional, português, como em inglês e espanhol. Foram utilizados como descritores isolados, ou combinados, para a busca em base de dados: "Endocrown", "Endocrown Prótese", "Endocrown Prosthesis", "Endocrown Prótesis" para compor a base temática da revisão e com um resultado de 31 artigos utilizados.

\section{RESULTADOS E DISCUSSÃO}

\section{- Aspectos importantes das endocrowns}

Segundo Dejak e Mtotkowski ${ }^{3}$, apesar das vantagens da utilização de pinos pré-fabricados, esse tipo de tratamento ainda continua tendo suas limitações devido à necessidade da retenção intrarradicular, podendo levar a uma perda de até
58,3\% da estrutura dentária. Segundo autores como Zavanelli et al. $^{11}$, as endocrowns podem ser classificadas como uma restauração indireta feita em monobloco cerâmico, ou seja, coroa e núcleo unidos, formando uma peça única com o mesmo material que terá uma retenção macro-mecânica no interior da câmara pulpar, promovendo estabilidade, juntamente com uma retenção micro-mecânica através da cimentação com materiais adesivos.

Para Carlos et al. ${ }^{5}$ a confecção desse tipo de peça protética pode ser realizada através do sistema CAD-CAM, garantindo resultado de alta compatibilidade e ótimas propriedades mecânicas, permitindo que se devolva a estética e função para o paciente e melhor adaptação marginal. Concordando com Carlos et al. ${ }^{5}$ os estudos de Rocca et al. ${ }^{12}$ avaliaram a adaptação marginal de molares tratados endodonticamente e restaurados com endocrown de resina composta, com ou sem reforço de pinos de fibra de vidro. Os resultados mostraram que não houve diferença significativa da adaptação marginal e o reforço não apresenta influência significativa.

Em um estudo coordenado por Gresnigt et al. ${ }^{13}$, os autores compararam a resistência a fratura da endocrown com os resultados dos dentes naturais sob forças axiais e laterais, para em seguida avaliar os tipos de falhas após os testes. Os dois grupos se mostraram vulneráveis aos dois tipos de cargas utilizados. Corroborando com Gresnight et al. ${ }^{13}$, em uma pesquisa feita por Rocca et al. ${ }^{14}$ foi avaliado o comportamento, a fadiga e o modo de fratura de endocrowns para pré-molares e nenhuma amostra apresentou falhas ou danos que pudessem ser detectados em microscópio estereoscópico.

De acordo com Fages e Bennasar ${ }^{15}$, para o preparo das endocrows é necessário que haja uma altura em extensão da câmara pulpar de pelo menos 3 $\mathrm{mm}$ para que não ocorra danos, como fratura. $\mathrm{Na}$ câmara pulpar são eliminadas as retenções das paredes deixando-as com uma expulsividade de 10 graus. É feita uma redução da altura oclusal de, no mínimo, $2 \mathrm{~mm}$. As margens do preparo devem permanecer supra gengivalmente afim de se obter uma cimentação adesiva de qualidade. Por outro lado, um estudo feito por Hayes et al. ${ }^{16}$ analisou o efeito da extensão da câmara pulpar do endocrown na resistência à fratura de molares mandibulares, e verificaram que essa extensão de $2 \mathrm{~mm}$ e $4 \mathrm{~mm}$ não garantem maior resistência à fratura e ao estresse. De forma menos positiva, Gaintantzopoulou e ElDamanhoury $^{17}$ concluíram que as extensões intrarradiculares das coroas endocrowns afetaram de forma negativa a adaptação marginal e ajuste interno da restauração.

A respeito do local de fraturas mediante forças oclusais, os resultados de Rocca et al. ${ }^{12} \mathrm{em}$ 2015 vão ao encontro aos estudos de 2016 de Rocca et al. ${ }^{14}$, mostrando que as fraturas ocorrem nos locais 
de maior força oclusal, no sentido corono-apical, propagando assim para a estrutura dental e rompendo abaixo da junção cemento-esmalte.

Conforme afirmam Rocca et al. ${ }^{14}$, apesar de estético e com módulo de elasticidade próxima a dos tecidos dentários, a endocrown ainda possui dificuldades em sua ligação dentro do canal radicular. A degradação de interface restaurada associada a diferenças na anatomia dos canais radiculares dificulta o controle da umidade. Rocca et al. ${ }^{14}$ ainda apontam que as tensões térmicas, os meios aquosos, as cargas oclusais mecânicas e os movimentos laterais, degradam a adesão da resina, apesar não ter sido tão significantes à presença da extensão da endocrown para dentro do canal radicular, comparado à coroa que só possuía ligação adesiva.

Baratieri et al. ${ }^{18}$ apontaram que as endocrowns apresentam maior longevidade em molares, pois em pré-molares a área da base disponível para adesão da prótese apresenta-se menor do que a altura da coroa, não trazendo assim bons resultados a longo prazo. Em contrapartida, Rocca et al. ${ }^{19}$ exploraram os limites de fadiga de endocrowns cerâmicos para pré-molares e evidenciaram que os núcleos mais longos exibiram resultados após fadiga equivalente às coroas tradicionais. Ou seja, a retenção melhorou e passou a não depender apenas da adesividade do cimento.

Contraditoriamente Sedrez-Porto et al. ${ }^{20} \mathrm{em}$ revisão sistemática da literatura observaram que os pré-molares e molares podem receber forças semelhantes durante a função, apresentando resultados semelhantes quando considera-se apenas os dentes posteriores. Ainda de acordo com SedrezPorto et al. ${ }^{20}$, as restaurações endocrowns possuem maior resistência à fratura quando comparadas com coroas totais acompanhadas de núcleo, restaurações em resina composta e inlays/onlays. Isso se deve à sua espessura oclusal que varia de 3 a $7 \mathrm{~mm}$, enquanto nas coroas convencionais esta espessura fica em torno de 1,5 a $2 \mathrm{~mm}$ apenas. Em geral, coroas convencionais são fabricadas a partir de diferentes materiais que possuem diferentes módulos de elasticidade fazendo com que este maior número de interfaces entre materiais distintos não distribua as tensões adequadamente, em contrapartida, a natureza monobloco das restaurações endocrown suportaria maior tensão por não apresentar diversas interfaces.

Um estudo realizado por Borgio Botto et al. ${ }^{21}$ teve como objetvo apresentar o resultado clínico retrospectivo de 11 endocrowns em sua prática privada, no período de 8 a 19 anos. No momento do exame, $10(90,9 \%)$ Endocrowns estavam na função e uma $(9,1 \%)$ havia falhado. Com o resultado desse estudo, concluiu-se que a endocrown é um procedimento restaurador tecnicamente sensível, conservador, estético, fácil, rápido, com funcionalidade e longevidade muito aceitável, para reabilitar dentes posteriores endodonticamente tratados, principalmente molares.

Para comparar as cargas de falha de dentes restaurados com endocrowns, coroas post-core sem ponteira, e coroas post-core com ponteira fornecidos com uma coroa depois de um alongamento, Rayyan et al. ${ }^{22}$ selecionaram 27 dentes molares inferiores, 1,5 $\mathrm{mm}$ acima da junção cemento seccionada (CEJ) e tratados endodonticamente. Pôde-se concluir que a fratura da raiz foi de modo dominante em falha em todos os grupos e que dentes molares restaurados com endocrowns têm resistência à fratura superior em comparação com aqueles restaurados com coroas retidas pós-núcleo.

Em uma análise crítica literatura feita por Silva e $\operatorname{Santos}^{23}$ verificaram que é abundante as pesquisas comparando o retentor intrarradicular fundido com outros pinos aplicados no dia a dia clínico. Já a comparação deste material com a endocrown não foi evidenciada nas buscas realizadas. Ainda afirmaram que é consenso na literatura científica que retentores intrarradiculares apenas promovem retenção do material restaurador, não promovendo reforço do remanescente dental. As estratégias de busca utilizadas não resultaram artigos que comparassem o comportamento biomecânico do retentor intrarradicular fundido e da endocrown, e também não alcançou registros que abordassem o uso de retentores intrarradiculares fundidos em dentes posteriores, necessitando de uma abordagem mais criteriosa para que os próximos estudos sejam utilizados termos mais delimitadores.

Outras características importantes para indicação segundo Biacchi et al. ${ }^{7}$, especificamente nos casos dos molares, seriam em casos dos mesmos com raízes pequenas, obliteradas, dilaceradas ou frágeis, pois nesses casos seria ainda mais complexo o uso de um retentor intrarradicular associado a uma coroa total tradicional. Concordando com esses fatos, Eldamanhoury et al. $^{24}$ afirmam que reabilitações extensas em molares tratados endodonticamente, apresentando canais atrésicos ou raízes curvas. Conforme afirma Chang et al. ${ }^{4}$, devido a esses fatores, pode-se explicar que existe um maior cuidado na indicação desse tipo de tratamento para pré-molares. A anatomia de dentes pré-molares confere uma maior dificuldade de adesão, com sua cúspide sendo mais alta, há uma maior força exercida nele levando a um possível movimento de alavanca no elemento dental. Dejak e Mtotkowski ${ }^{3}$ acrescentam que, da mesma forma que os prémolares, a indicação de endocrowns para incisivos pode ser comprometida devido à altura comparada com a largura, às forças mastigatórias aplicadas podem levar a um movimento de alavanca sobre o dente, resultando em uma possível fratura.

Por esta razão, segundo Zavanelli et al. ${ }^{11}$, a interface do dente no tamanho adequado para que 
uma endocrown funcione é muito importante, devido a isso, em molares cabe a melhor indicação, pois a sua interface é suficiente para que se evitem movimentos de alavanca.

Conforme afirma Rocca et al. ${ }^{1}$, inúmeras são as vantagens para o uso da técnica endocrown como o restabelecimento estético e função, permitindo maior conservação de tecido dentinário e esmalte periférico em comparação a coroas totais convencionais, visto que essa preservação de tecido promove maior estabilidade marginal.

Segundo Carlos et al. ${ }^{5}$, em relação à confecção da peça protética, com a evolução dos sistemas cerâmicos e dos sistemas de confecção, temos vantagens de se obter uma fresagem mais precisa, com formato personalizado, adaptação da superfície interior da restauração e a melhor replicação da morfologia oclusal, as imprecisões e contaminação cruzada são mínimas, evita contração de polimerização por ser uma restauração indireta e se tem melhor adesão e satisfação do paciente. Em relação aos passos técnicos, Zavanelli et al. ${ }^{11}$ dizem ainda que os mesmos sejam diminuídos em comparação aos passos de quando é realizada uma reabilitação com núcleo ou pino com coroas totais, portanto, menos sessões clínicas são requisitadas.

Outro ponto importante destacado por Zavanelli et al. ${ }^{11}$ seriam as margens da restauração são mantidas fora do periodonto, sendo assim benéfico para conservação da higiene oral e saúde periodontal. Mesmo assim, a instrução ao paciente em relação à adequada higiene bucal é de suma importância para que auxilie ainda mais no sucesso do tratamento. Baratieri et al. ${ }^{18}$ complementa os fatores elencados por Zavanelli et al. ${ }^{11}$ afirmando que sobre microinfiltração marginal o sucesso de uma restauração endocrown está relacionado diretamente à qualidade da adesão tanto à peça quanto ao substrato dental.

Para Valentina et al. $^{25}$, as principais desvantagens das coroas endodônticas adesivas seriam a perda de retenção e fratura relacionada ao tamanho e forma do canal radicular e/ou câmara pulpar e ao material utilizado em suas extensões intrarradiculares. Por outro lado, Sedrez-Porto et. al. ${ }^{20}$ afirmaram em sua revisão sistemática que o motivo principal de falha em endocrowns foram cáries secundárias, mas disseram que nenhum estudo citou perda de retenção ou fratura da restauração.

A utilização de um material resistente para a confecção da endocrown é importante, pois, as cargas mastigatórias incidentes sobre a coroa são consideráveis. Atualmente, isso é possível graças à evolução dos materiais destinados para este fim, tais como as cerâmicas odontológicas. De acordo com Gresnigt et al. ${ }^{13}$, a resina multifásica tem sido defendida como uma alternativa para cerâmica reforçada por ter modo de elasticidade e propriedades semelhantes à estrutura dental, apesar disso, o autor afirma existir pouca informação sobre sua durabilidade.

Em um estudo com 30 molares extraídos feito por Gaintantzopoulou e El-Damanhoury ${ }^{17}$, foi comparada a resistência à fratura de endocrowns fabricadas de diferentes materiais: cerâmica feldspática, dissilicato de lítio e resina composta. Concluiu-se que o material mais resistente à fratura seria a resina, porém este também seria o material que mais causaria infiltração marginal.

Em relação ao material utilizado, deve-se evitar algum material com uma diferença muito grande entre o módulo de elasticidade em comparação com a dentina. Deve ser considerado qual o dente será reabilitado para não haver intercorrências devido à resistência flexural da peça. Em um caso clínico apresentado por Zavanelli et al. ${ }^{11}$ foi escolhido o dissilicato de lítio monolítico que possui resistência flexural de $400 \mathrm{MPa}$ (Mega Pascal), que é suficiente para aguentar as cargas de forças de molares, além de que esse material tem uma estética muito satisfatória devido a sua composição que possui uma matriz vítrea, sendo classificada como uma cerâmica ácido-sensível.

Carlos et al. ${ }^{5}$ vai ao encontro a Zavanelli et al. ${ }^{11}$ afirmando que os materiais de escolha para confecção da endocrown são as cerâmicas ácidosensível como, por exemplo, o dissilicato de lítio reforçado por fibra, que dentro dessa classificação seria uma cerâmica mais resistente, e com o reforço das propriedades de absorção de tensões, manteria uma estética desejável.

Tysowsky $^{26}$ concorda que o material mais indicado para a fabricação das endocrowns ainda vem sendo as cerâmicas reforçadas por dissilicato de lítio, usinadas pelo sistema CAD/CAM. Corroborando com este fato, Qin et al. ${ }^{27}$ afirmam que o dissilicato de lítio é uma cerâmica condicionável que, por sua vez, possui excelente aderência com a estrutura dentária, resistência mecânica, altas propriedades estéticas e maior longevidade, desta forma, o tipo de material escolhido tem papel fundamental na determinação do sucesso no desempenho das endocrowns.

Entretanto, Biacchi et al. ${ }^{7}$ relataram que o material de escolha mais adequado seria uma cerâmica ácido-resistente tal como a zircônia, pois garante a resistência mecânica necessária e adequada para suportar as forças oclusais e de resistência entre restauração e paredes da cavidade. Dessa forma, não existe um consenso a respeito material mais indicado para essas situações.

Corroborando com Biacchi et al. ${ }^{7}$, Zavanelli et al. ${ }^{11}$ afirmam que a zirconia é uma cerâmica que também pode ser indicada, possui uma resistência flexural que varia de 900 a $1.200 \mathrm{MPa}$, porém devido ser classificada como uma cerâmica ácido-resistente, 
ou seja, sem essa matriz vítrea, sua estética não é favorável, sendo uma cerâmica extremamente branca e opaca.

Uma pesquisa realizada por El Ghoul et al. ${ }^{28}$ teve o objetivo de avaliar in vitro a resistência à fratura e os modos de falha de endocrowns feitos de três materiais (cerâmica de vidro de dissilicato de lítio, cerâmica de vidro de silicato de lítio reforçada com zircónio e nano-cerâmica de resina) de projeto assistido por computador / CAD (Computer-Aided Manufacturing - CAD / CAM) sujeitos a carga ciclável termo-mecânica. A vitrocerâmica de dissilicato de lítio registrou a maior resistência à fratura sob carga axial e lateral. O número de falhas irreparáveis com todos os materiais de endocrown testados ainda não sugere o uso deste tipo de restaurações em dentes posteriores.

Um estudo realizado por El Ghoul et al. ${ }^{29}$ objetivou avaliar in vitro o efeito de materiais à base de resina e cerâmica sobre a adaptação interna e marginal de endocrowns, tais como: grupo $\mathrm{C}$ : nanocerâmica híbrida (Cerasmart; GC Corp), grupo T: material compósito de fibra (Trilor; Bioloren Srl), grupo E: vitrocerâmica de dissilicato de lítio (IPS e.max CAD; Ivoclar Vivadent $A G$ ) e grupo V: vitrocerâmica de silicato de lítio reforçada com zircónio (Vita Suprinity; VITA Zahnfabrik GmbH). Verificaram que todos os materiais apresentaram intervalos internos e marginais clinicamente aceitáveis, exceto para o gap marginal do grupo compósito de fibra (Trilor).

Valentina et al. ${ }^{25}$ também afirmam que é possível usar o sistema CAD/CAM, que trabalha com uma maior gama de materiais e possui como vantagem a automatização da fabricação da peça e consequente melhor qualidade da mesma, diminuindo a chance de erros humanos e utilizando única sessão clínica. Uma desvantagem seria a utilização de blocos monocromáticos, utilizando pigmentação externa para se atingir a estética, porém o surgimento de blocos com três cores tem uma passagem promissora para a parte estética das restaurações fresadas.

Um estudo feito por Zou et al. ${ }^{30}$ para avaliar clinicamente o design auxiliado por computador (CAD/CAM), após 6 meses e 1,2 e 3 anos de serviço clínico notaram que nenhuma das 289 endocrowns realizadas falhou durante o período de observação. Os altos critérios de classificação clínica $(97,2 \%)$ e o alto percentual de satisfação $(98,0 \%)$ permaneceram praticamente inalterados ao longo das avaliações aos 6 meses e após 1, 2 e 3 anos. As restaurações de endocrown com zircônia monolítica representam um método eficaz de restaurar os dentes posteriores após a terapia do canal radicular. Com base no período de observação de curto prazo do presente estudo, as endocrowns de zircônia monolítica podem ser consideradas uma restauração confiável para molares tratados endodonticamente com extensa perda de substância coronária.

Outro ponto de extrema importância, além da escolha do material é o processo de cimentação da endocrown. Para que seja conferida a retenção micromecânica deve-se lançar mão de um material cimentante adesivo, sendo compatível com a cerâmica utilizada. No caso clínico também apresentado por Zavanelli et al. ${ }^{11}$ o cimento resinoso utilizado foi o Variolink N (Ivoclar), que confere um sistema dual de polimerização, tanto fotoativado, quanto quimicamente ativado.

Essa característica do cimento dual é importante, pois, devido à retenção macromecânica ser no interior da câmara pulpar, ao utilizar o fotopolimerizador, a luz não consegue atravessar totalmente a própria cerâmica para chegar nessa região, promovendo uma deficiência na cimentação. Dessa forma o sistema dual, dribla essa dificuldade, promovendo uma polimerização química que independe de luz para essa região mais dificultosa e a polimerização através da foto ativação auxilia nesse processo, agindo principalmente na linha de cimentação ${ }^{11}$.

\section{CONCLUSÃO}

Diante do exposto, a alternativa restauradora Endocrown sob boa indicação e avaliação assídua do caso clínico, é possível obter previsibilidade de sucesso, e às vezes até melhor opção que os retentores intrarradiculares. Logo, indicada para dentes posteriores tratados endodonticamente, confeccionada na maioria das vezes por dissilicato de lítio e apresentar estudos que comprovem que o risco de fratura ser inferior às reabilitações com uso de retentores, ainda são necessárias mais pesquisas para comprovar cada vez mais a eficácia dessa alternativa.

\section{REFERÊNCIAS}

1. Rocca GT, Rizcalla N, Krejci I. Fiber-reinforced resin coating for endocrown preparations: a technical report. Oper Dent. 2013;38(3):242-48.

2. Borges Junior HE, Sábio S, Bender KRF, Costa YM, Mondelli J. Endocrown - avaliação da resistência dos cimentos dentários. Rev Odontol Araçatuba. 2013;34(2):23-6.

3. Dejak B, Młotkowski A. 3D-Finite element analysis of molars restored with endocrowns and posts during masticatory simulation. Dent Mater. 2013;29(12):e309-17.

4. Chang CY, Kuo JS, Lin YS, Chang YH. Fracture resistance and failure modes of CEREC endocrowns and conventional post and core-supported CEREC crowns. J Dent Sci 2009;4(3):110-17.

5. Carlos RB, Thomas Nainan M, Pradhan $S$, Sharma R, Benjamin S, Rose R. Restoration of endodontically treated molars using all ceramic endocrowns. Cape Rep Dent. 2013; 2013:210763. 
6. Magne P, Knezevic A. Simulated fatigue resistance of composite resin versus porcelain $\mathrm{CAD} / \mathrm{CAM}$ overlay restorations on endodontically treated molars. Quintessence Int. 2009;40(2):125-33.

7. Biacchi GR, Mello B, Basting RT. The endocrown: an alternative approach for restoring extensively damaged molars. J Esthet Restor Dent. 2013;25(6):383-90.

8. Dietschi D, Duc O, Krejci I, Sadan A. Biomechanical considerations for the restoration of endodontically treated teeth: a systematic review of the literature, Part II (Evaluation of fatigue behavior, interfaces, and in vivo studies). Quintessence Int. 2008;39(2):117-29.

9. Mörmann WH, Bindl A, Lüthy H, Rathke A. Effects of preparation and luting system on allceramic computer-generated crowns. Int J Prosthodont. 1998;11(4):333-39.

10. Bernardo WM, Nobre MRC, Jatene FB. A prática clinica baseada em evidências. Parte II: buscando as evidências em fontes de informação. Rev Assoc Med Bras. 2004;50(1):104-8.

11. Zavanelli AC, Zavanelli RA, Mazaro JVQ, Lemos CAA, Dias ES, Silva EV, Pinto RC. Coroas Endoncrown: uma revisão de literatura e relato de caso. Arch Health Invest. 2017;6(8):382-89.

12. Rocca GT, Saratti CM, Cattani-Lorente M, Feilzer AJ, Scherrer S, Krejci I. The effect of a fiber reinforced cavity configuration on load bearing capacity and failure mode of endodontically treated molars restored with $\mathrm{CAD} / \mathrm{CAM}$ resin composite overlay restorations. J Dent. 2015; 43(9):1106-15.

13. Gresnigt MM, Özcan M, van den Houten ML, Schipper L, Cune MS. Fracture strength, failure type and Weibull characteristics of lithium disilicate and multiphase resin composite endocrowns under axial and lateral forces. Dent Mater. 2016;32(5):607-14.

14. Rocca GT, Sedlakova P, Saratti CM, Sedlacek R; Gregor L; Rizcalla $\mathrm{N}$ et al. Fatigue behavior of resin-modified monolithic CAD-CAM RNC crowns and endocrowns. Dent Mater. 2016;32(12):e338-50.

15. Fages M, Bennasar B. The endocrown: a different type of all-ceramic reconstruction for molars. J Can Dent Assoc. 2013;79:d140.

16. Hayes A, Duvall N, Wajdowicz M, Roberts H. Effect of Endocrown Pulp Chamber Extension Depth on Molar Fracture Resistance. Oper Dent. 2017;42(3):327-34.

17. Gaintantzopoulou MD, El-Damanhoury HM. Effect of Preparation Depth on the Marginal and Internal Adaptation of Computer-aided Design/Computer-assisted Manufacture Endocrowns. Oper Dent. 2016;41(6):607-16.

18. Baratieri LN, Monteiro S JR. Coroas posteriores endocrown. In: Baratieri L N. Odontologia restauradora: fundamentos e técnicas, São Paulo: Santos, 2010. v. 2, cap.30, p.738-55.

19. Rocca GT, Saratti CM, Poncet A, Feilzer AJ, Krejci I. The influence of FRCs reinforcement on marginal adaptation of CAD/CAM composite resin endocrowns after simulated fatigue loading. Odontology. 2016;104(2):220-32.

20. Sedrez-Porto JA, Rosa WL, da Silva AF, Münchow EA, Pereira-Cenci T. Endocrown restorations: A systematic review and metaanalysis. J Dent. 2016;52:8-14.

21. Borgia Botto E, Barón R, Borgia JL. Endocrown: Estudio clínico retrospectivo de una serie de pacientes, en un período de 8 a 19 años. Odontoestomatología 2016;18(28):48-59.

22. Rayyan MR, Alauti RY, Abanmy MA, AlReshaid RM, Bin Ahmad HA. Endocrowns versus postcore retained crowns for restoration of compromised mandibular molars: an in vitro study. Int J Comput Dent. 2019;22(1):39-44.

23. Silva ACC, Santos FB. Opções protéticas para dentes posteriores tratados endodonticamente: revisão de literatura [monografia]. Lagarto - SE: Departamento de Odontologia de Lagarto da Universidade Federal de Sergipe - UFS; 2018.

24. El-Damanhoury HM, Haj-Ali RN, Platt JA. Fracture resistance and microleakage of endocrowns utilizing three CAD-CAM blocks. Oper Dent. 2015;40(2):201-10.

25. Valentina V, Aleksandar T, Dejan L, Vojkan L. Restoring endodontically treated teeth with allceramic endo-crowns - case report. Serbian Dent J 2008;55:54-64.

26. Tysowsky GW. The science behind lithium disilicate: a metal-free alternative. Dent Today. 2009;28(3):112-13.

27. Qin F, Zheng S, Luo Z, Li Y, Guo L, Zhao Y et al. Evaluation of machinability and flexural strength of a novel dental machinable glassceramic. J Dent. 2009;37(10):776-80.

28. El Ghoul W, Özcan M, Silwadi M, Salameh Z. Fracture resistance and failure modes of endocrowns manufactured with different CAD/CAM materials under axial and lateral loading. J Esthet Restor Dent. 2019;31(4):378-87.

29. El Ghoul WA, Özcan M, Ounsi H, Tohme H, Salameh Z. Effect of different CAD-CAM materials on the marginal and internal adaptation of endocrown restorations: An in vitro study. $\mathrm{J}$ Prosthet Dent. 2020;123(1):128-34.

30.Zou Y, Bai J, Xiang J. Clinical performance of CAD/CAM-fabricated monolithic zirconia endocrowns on molars with extensive coronal loss of substance. Int J Comput Dent. 2018;21(3):225-32. 


\section{CONFLITO DE INTERESSES}

Os autores declaram não haver conflitos de interesse.

\section{AUTOR PARA CORRESPONDÊNCIA}

José Henrique de Araújo Cruz

Rua Paulo Diogenes, 57, Centro,

59990-000 Rafael Fernandes/RN, Brasil

Telefone: (83) 99625-0125

E-mail: henrique_araujo1992@hotmail.com

Submetido em 17/06/2019

Aceito em 20/04/2020 